\title{
Molecular Simulation of Loading Dependent Slow Diffusion in Confined Systems
}

\author{
E. Beerdsen, ${ }^{*}$ B. Smit, and D. Dubbeldam \\ Department of Chemical Engineering, University of Amsterdam, Nieuwe Achtergracht 166, \\ 1018 WV Amsterdam, The Netherlands
}

(Received 23 April 2004; revised manuscript received 16 July 2004; published 6 December 2004)

An extension to transition state theory is presented that is capable of computing quantitatively the diffusivity of adsorbed molecules in confined systems at nonzero loading. This extension to traditional transition state theory yields a diffusivity in excellent agreement with that obtained by conventional molecular dynamics simulations. While molecular dynamics calculations are limited to relatively fast diffusing molecules or small rigid molecules, our approach extends the range of accessible time scales significantly beyond currently available methods. It is applicable in any system containing free energy barriers and for any type of guest molecule.

DOI: 10.1103/PhysRevLett.93.248301

The adsorption and diffusion of molecules in confined systems is of great importance to many industrial processes such as the separation of linear and branched alkanes. The performance of confinements in separation and catalytic processes depends critically on the match between the confinement and the shape and the size of the adsorbate [1]. Because diffusion is the rate limiting factor in many catalytic processes, diffusion in systems like zeolites has been widely studied [2-9]. However, in contrast to adsorption and separation, the diffusion of molecules in tight confinement is not yet well understood.

One of the difficulties encountered when studying diffusion behavior is that many processes occur outside the time scale accessible to molecular dynamics (MD), which is typically limited to diffusion rates on the order of $10^{-12} \mathrm{~m}^{2} / \mathrm{s}$. To overcome this, some studies have used dynamically corrected transition state theory (dcTST) methods [10-13]. Hitherto, studies were limited to the infinite dilution limit, whereas many of the processes of practical importance occur at nonzero loading. Coarsegrained kinetic Monte Carlo (KMC) studies have pointed at the difficulties in taking into account the various correlations induced by particle-particle interactions $[10,14]$. In this Letter we resolve this problem by extending dcTST to include diffusion of molecules at nonzero loading. We show that these correlations can be taken into account by a proper definition of an effective hopping rate of a single particle. This hopping rate can be computed accurately using rare-event simulation techniques at the conditions of interest.

A suitable and well-studied system to study diffusion in confinement is the LTA-type zeolite shown in Fig. 1. The system consists of cubically arranged cages of about $10 \AA$ in size, where each cage has 15 and 12 distinct adsorption sites for methane and ethane, respectively. The cages are connected by narrow windows that form large free energy barriers. For small molecules, the positions in the window regions are favorable adsorption sites, and the windows form entropic, not energetic, barriers. An advantage of this system is that studying diffusion of
PACS numbers: 66.30.-h, 47.55.Mh, 68.43.Jk, 82.75.Jn

small molecules with MD is still feasible, and this allows a detailed comparison with our new approach.

In this system, diffusion can be considered an activated process, in which the particle hops from one cage to the next, and the actual crossing time is negligible compared to the time a particle spends inside the cage. One can exploit the large separation in time scales using rareevent simulation techniques. We consider a system which can be in two stable states, $A$ and $B$, with a dividing free energy barrier between them. We define a reaction coordinate $q$, which indicates the progress of the diffusion event from cage $A$ to cage $B$, as the Cartesian coordinate along the axis parallel to the line connecting the center points of $A$ and $B$. The location of the dividing barrier is denoted by $q^{*}$ (see Fig. 1). In the Bennett-Chandler approach [15-17] one computes the hopping rate over the barrier in two steps. First, the relative probability $P\left(q^{*}\right)$ is computed to find a particle on top of the barrier, given that it is in state $A$, and subsequently the averaged veloc-
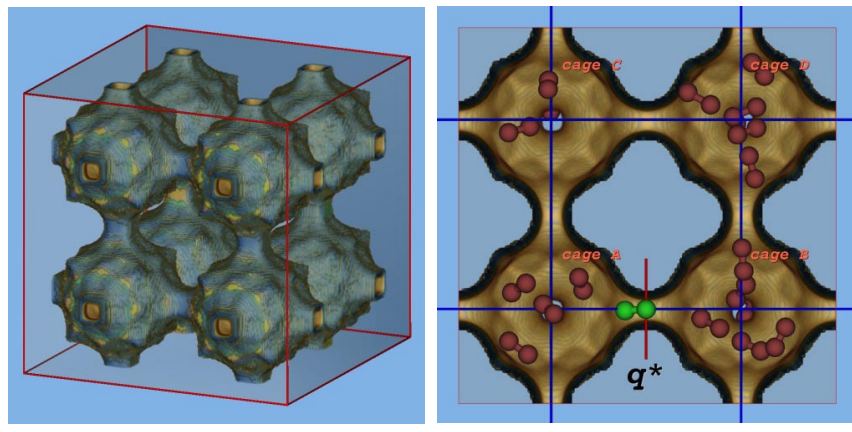

FIG. 1 (color online). (left) A unit cell of the LTA-type zeolite. The dimensions of the cubic unit cell are $24.555 \AA$. It contains eight cages connected in a cubic arrangement and each cage is connected to six other cages by windows of about $5 \AA$ in diameter. (right) Typical snapshot of ethane $\left(\mathrm{CH}_{3}-\right.$ $\mathrm{CH}_{3}$ ) at an average loading of four molecules per cage at $750 \mathrm{~K}$, constraining one tagged molecule at the dividing surface $q^{*}$. The hopping events are coarse grained on a lattice spanned by the cage centers. 
ity at the top of the barrier $\sqrt{k_{B} T / 2 \pi m}$ (assuming that the particle velocities follow a Maxwell-Boltzmann distribution) and the probability $\kappa$ that the system ends up in state $B$. The transmission rate $k_{A \rightarrow B}$ from cage $A$ to cage $B$ is then given by

$$
\begin{gathered}
k_{A \rightarrow B}=\kappa \times \sqrt{\frac{k_{B} T}{2 \pi m}} \times P\left(q^{*}\right), \\
P\left(q^{*}\right)=\frac{e^{-\beta F\left(q^{*}\right)}}{\int_{\operatorname{cage} A} e^{-\beta F(q)} d q},
\end{gathered}
$$

where $\beta=1 /\left(k_{B} T\right), k_{B}$ is the Boltzmann constant, $T$ is the temperature, $m$ is the mass involved in the reaction coordinate, and $F(q)$ is the free energy as a function of $q$. In first order approximation, TST assumes that all particles that reach the barrier with a velocity towards $B$ do end up in $B$, i.e., $\kappa=1$.

In dcTST, the transmission coefficient $\kappa$ corrects for recrossing events; i.e., it corrects for trajectories which cross the transition state from $A$ but fail to end up in $B$. In general, the reaction coordinate $q$ is a function of the configuration of the whole system, i.e., $q=q\left(\mathbf{r}_{1}, \ldots, \mathbf{r}_{N}\right)$. However, we can choose $q$ as the position of one of the atoms of the diffusing molecules [11]. This choice of order parameter underestimates the free energy of the true transition state, but the dynamical correction $\kappa$ is the exact correction compensating our choice of reaction coordinate [16]. The transmissions are fast events and can be computed using MD as the fraction of particles coming from the initial state $A$ that successfully reaches the final state $B$ out of those that cross the dividing surface at $t=0$. The transmission coefficient reaches a clear plateau value as a function of time, indicating all short time scale recrossings have been eliminated.

In the limit of infinite dilution there are no interparticle correlations and the particles perform a random walk on a lattice spanned by the cage centers. The transmission rates are then easily converted to self-diffusion coefficients by

$$
D_{S}=k_{A \rightarrow B} \lambda^{2}=\frac{1}{6} k \lambda^{2},
$$

with $\lambda$ the center-to-center lattice distance of the LTA cages $(12.2775 \AA)$. Because we calculate the hopping rate from $A$ to $B$ in one direction only, $k_{A \rightarrow B}=1 / 6 k$.

The extension of dcTST to finite loading is nontrivial. Conventional methods use a hierarchical approach to compute elementary hopping rates for use in a subsequent KMC scheme to obtain self-diffusion and collective diffusion coefficients $[2,3,13,14,18]$. Let us consider the class of cage/window-type systems (e.g., methane in LTA) where the barriers are entropical in nature. At nonzero loading a molecule hopping from $A$ to $B$ induces a vacancy. The vacancy induces an increased probability of particles to hop to cage $A$. These correlated jumps may significantly influence the hopping process and should be included in order to obtain a correct diffusion coefficient. In a KMC simulation, the surrounding particles remain in their fixed positions (no two jumps can occur at the same time) and this constraint suppresses these correlations. We are not aware of a KMC scheme that takes into account these simultaneous jumps.

We take a different viewpoint on computing diffusivities in such systems. The correlations can be taken into account by a proper definition of an effective hopping rate of a single particle. We compute the self-diffusion coefficient directly. This is done by computing the hopping rate of a molecule over a typical length scale $\lambda$ given by the smallest repeating zeolite structure (i.e., from the center of cage $A$ to the center of cage $B$, implicitly integrating over all adsorption sites in the cage, irrespective of whether these are well defined or not). The other particles are regarded as a contribution to the external field exerted on the tagged particle. Since we look at a single tagged particle, the diffusion coefficient can still be computed from the hopping rate by using Eq. (3) at any loading, rendering it unnecessary to perform $N$-particle KMC simulations. Now, $k_{A \rightarrow B}$ is the effective hopping rate, including all jump correlations and averaged over all orientations and loading fluctuations. The external field is maintained by an MC NVT simulation (fixed total number of particles, volume, and temperature) in the "background." By using an MC approach that includes translational, orientational, and regrow moves, we automatically average over cage distributions, positions, and orientations of neighboring molecules. To speed up these simulations for longer molecules by several orders of magnitude, these techniques can be combined with the configurational bias Monte Carlo approach [17].

The proposed method relies on the direct inclusion of all interparticle correlations in the effective hopping rate of a particle traveling from cage $A$ to cage $B$. In our calculations, we have observed that to obtain agreement with MD results, one cannot limit the free energy calculation to the two cages $A$ and $B$ for which the hopping is computed. It is essential to average over fluctuations in the number of particles in the neighboring cages. By "closing off" cages, the system is intrusively changed. Figure 2 (top right) compares the correct free energy (very large simulation box with on average of eight molecules per cage), with approximations by simulating a fixed number of eight particles in cage $A$ only, $A$ and $B$ only, and simulations with a fixed number of exactly eight particles in each cage. These small differences in the free energies result in diffusion coefficients that deviate up to $60 \%$. If we surround cage $A$ with one shell of neighboring cages, we obtain results that are identical to those obtained in the very large system. Inclusion of a second ring of cages is not necessary, as jump correlations over distances larger than two cages vanish. A similar influence is 

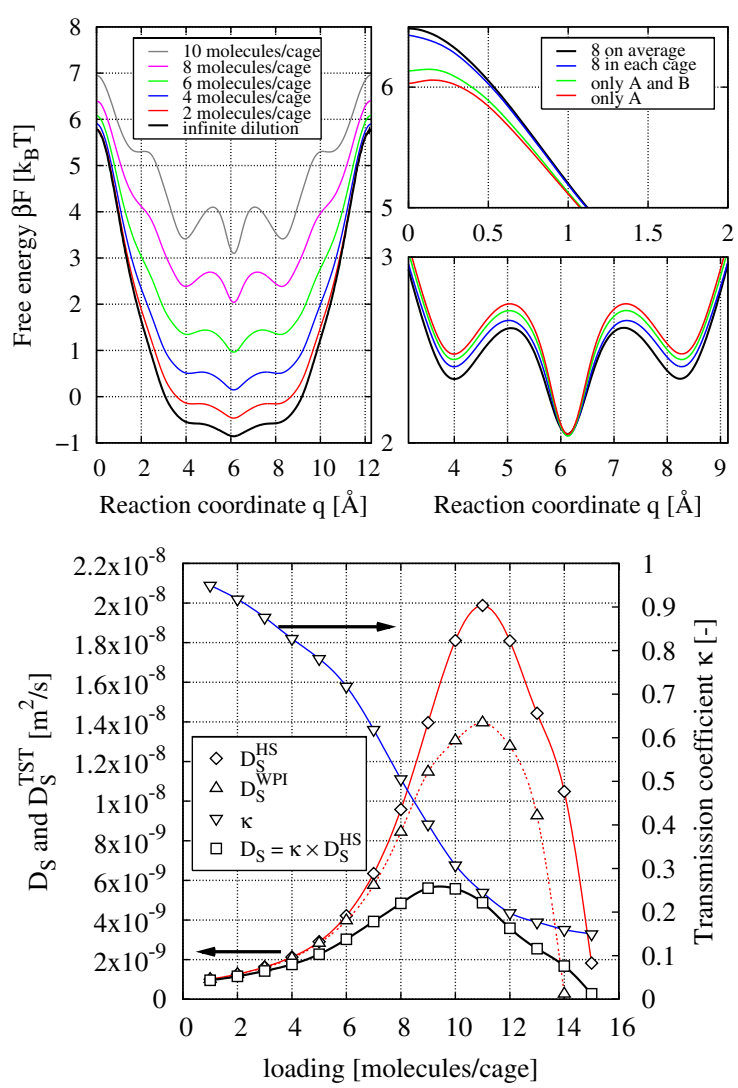

FIG. 2 (color online). (top left) Free energy profiles of methane in LTA at $600 \mathrm{~K}$ for various loadings, obtained using the HS method. (top right) Details from free energy profiles for eight molecules per cage, using different environments. (bottom) $D_{S}^{\mathrm{HS}}, D_{S}^{\mathrm{WPI}}$, and $D_{S}$ (left axis) and transmission coefficients (right axis) as a function of loading.

observed in the calculation of the transmission coefficient. Successful hopping events may induce a chain of hops of other particles, and this can influence the transmission coefficient. Only at low loadings we obtain agreement with MD.

We now discuss the two steps in the computation of the hopping rate using our approach in detail.

The probability $P(q)$. - During an NVT-ensemble MC simulation at the required loading we measure the free energy $F(q)$ by using either the Widom particle insertion (WPI) method or histogram sampling (HS). WPI uses a probe particle that is inserted at random positions to measure the energy required for or obtained by insertion of the particle in the system. This energy is mapped onto the reaction coordinate $q$, using $\beta F(q)=-\ln \left\langle e^{-\beta \Delta U}\right\rangle_{N}$, to produce a free energy profile, where $\left\langle e^{-\beta \Delta U}\right\rangle_{N}$ is the average Boltzmann factor over all positions in the slice perpendicular to the reaction coordinate. A "ghost particle" is used as the measuring probe, but the other particles in the system do not feel its presence. In the HS method, a histogram is made of the particle positions, mapped on the reaction coordinate. From the histogram a free energy profile is computed, by using $\beta F(q)=$ $-\ln \langle P(q)\rangle$. If needed, statistics can be improved by using importance sampling [17]. At higher loadings, WPI is known to give erroneous results [17]. In Fig. 2 (top left) we have plotted the free energy profiles as obtained from the HS method, for various loadings, and in Fig. 2 (bottom) the $D_{S}^{\mathrm{HS}}$ and $D_{S}^{\mathrm{WPI}}$ as a function of loading. At loadings as low as six methane molecules per cage the WPI method starts to deviate.

The transmission coefficient $\kappa$. - We compute the fraction of particles starting on top of the barrier with a velocity towards $B$ that successfully reach cage $B$. Starting configurations are generated using MC with one particle constrained to the dividing surface and $N-1$ particles moving around freely [see Fig. 1 (right)]. These configurations are then used to compute the ratio in unconstrained $N V E$-MD simulations, starting with velocities sampled from a Maxwell-Boltzmann distribution at the desired temperature. For this snapshot cage $B$ contains more molecules than cage $A$, and the barrier molecule has a high probability of recrossing to cage $A$. In general, the transmission coefficient is much lower than one for chain molecules (even at infinite dilution). Note that during the computation none of the windows are blocked and simultaneous jumps (e.g., from cage $C$ to cage $A$, and cage $D$ to cage $B$ ) are allowed.

Figure 2 (bottom) shows the individual components of the diffusion process, $D_{S}^{\mathrm{TST}}$ and $\kappa$, as a function of loading for methane in LTA. Although the transmission coefficient shows a monotonic decrease with density, the diffusion coefficient goes through a maximum. The driving force behind the initial increase in diffusion is a loss of guest-host attraction inside the cages. This interaction is being replaced by less favorable interparticle interaction, causing an increase of the free energy in the cage regions and thus a net decrease of the free energy barrier [Fig. 2 (bottom)]. Eventually, the free energy barrier increases again, due to packing and free-volume effects, causing a decrease of the diffusion coefficient. While the transmission coefficient only slightly changes the qualitative behavior of the diffusion as a function of loading, it has a profound quantitative influence [Fig. 2 (bottom)].

To validate our method, we show the diffusion in LTA of methane at $600 \mathrm{~K}$ and ethane at $750 \mathrm{~K}$ using both MD and extended dcTST. In addition, we show the diffusion of propane at $600 \mathrm{~K}$, using only dcTST, for which the diffusion is too slow to compute with MD. The LTA-type system used here is a cation-free version of the commonly used LTA 5A zeolite (four $\mathrm{Na}^{+}$and four $\mathrm{Ca}^{+}$per cage). The system size was a cubic box of $24.555 \AA$, containing eight cages in total. We used a united-atom model [19], in which we consider $\mathrm{CH}_{x}$ groups as single interaction centers with their own effective Lennard-Jones potentials. We used the position of the $\mathrm{CH}_{4}$ group, one of the $\mathrm{CH}_{3}$ groups, and the middle $\mathrm{CH}_{2}$ group as the dcTST reaction 


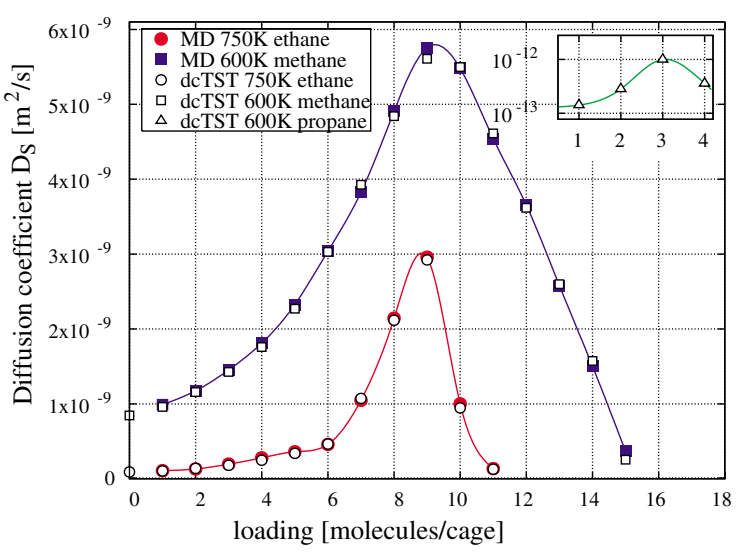

FIG. 3 (color online). Diffusion of methane, ethane, and propane in LTA, as a function of loading, at 600, 750, and $600 \mathrm{~K}$, respectively, computed by extended dcTST (HS method) and MD.

coordinate for methane, ethane, and propane, respectively [11]. The interactions between the rigid framework and the guest molecules are assumed to be dominated by the oxygen atoms [20]. The potential parameters are optimized to reproduce adsorption properties in pure silica confinements [21,22]. In the MD simulations we used a time step of $0.5 \mathrm{fs}$ with the velocity-Verlet integration scheme. The $N V T$ ensemble was imposed using a Nosé-Hoover thermostat. The duration of the computation was such that the error bars are smaller than the symbol size. As is shown in Fig. 3, our extended dcTST method and MD agree quantitatively. The presented methodology provides a general framework for computing diffusivities of molecules in systems where diffusion is sufficiently slow, due to free energy barriers, irrespective of whether these are energetical or entropical in nature. Longer molecules are efficiently handled and likewise, diffusion in mixtures can easily be computed; any type of particle can be considered part of the external field. A quantitative comparison with PFG (pulsed-field gradient)-NMR experimental results requires including the ions in the simulations. Beerdsen et al. have extended the united-atom model with cations [23], and our dcTST method already includes the necessary tools.

In summary, our method applies dcTST at nonzero loadings without introducing assumptions not already present in traditional TST methods. It can be used to explain diffusion behavior as a function of loading in any system with enough energy dissipation between hops, so that random walk theory (the assumption of equilibration between two subsequent jumps) and TST are valid, as we show here for alkanes in LTA. The method gives results in excellent agreement with MD but is also applicable in the regime of very slow diffusion where MD cannot be used. This extends the range of accessible time scales significantly beyond currently available methods.
Furthermore, the method enables us to express loading effects in terms of free energy differences.

We thank Professor S. M. Auerbach and Dr. T. J.H. Vlugt for their comments on the manuscript, the Netherlands Research Council (CW) and the Deutsche Forschungsgemeinschaft (DFG, priority program SPP 1155 ) for financial support, and NWO/NCF for computational resources.

*Corresponding author.

Electronic address: beerdsen@science.uva.nl

[1] J. M. Thomas, Sci. Am. 266, No. 4, 112 (1992).

[2] C. Saravanan, F. Jousse, and S. M. Auerbach, Phys. Rev. Lett. 80, 5754 (1998).

[3] E. J. Maginn, A.T. Bell, and D. N. Theodorou, J. Phys. Chem. 100, 7155 (1996).

[4] K. Hahn, J. Karger, and V. Kukla, Phys. Rev. Lett. 76, 2762 (1996).

[5] H. Jobic, J. Karger, and M. Bee, Phys. Rev. Lett. 82, 4260 (1999).

[6] L. A. Clark, G. T. Ye, and R. Q. Snurr, Phys. Rev. Lett. 84, 2893 (2000).

[7] D. S. Sholl and K. A. Fichthorn, Phys. Rev. Lett. 79, 3569 (1997).

[8] S. Fritzsche, M. Gaub, R. Haberlandt, and G. Hofmann, J. Mol. Model. 2, 286 (1996).

[9] E. B. Webb, G. S. Grest, and M. Mondello, J. Phys. Chem. B 103, 4949 (1999).

[10] S. M. Auerbach, Int. Rev. Phys. Chem. 19, 155 (2000).

[11] D. Dubbeldam, S. Calero, T. L. M. Maesen, and B. Smit, Phys. Rev. Lett. 90, 245901 (2003).

[12] F. Jousse and S. M. Auerbach, J. Chem. Phys. 107, 9629 (1997).

[13] R. L. June, A. T. Bell, and D. N. Theodorou, J. Phys. Chem. 95, 8866 (1991).

[14] C. Tunca and D. M. Ford, Chem. Eng. Sci. 58, 3373 (2003).

[15] C. H. Bennett, in Diffusion in Solids: Recent Developments, edited by A. Nowick and J. Burton (Academic Press, New York, 1975), pp. 73-113.

[16] D. Chandler, J. Chem. Phys. 68, 2959 (1978).

[17] D. Frenkel and B. Smit, Understanding Molecular Simulation (Academic Press, London, 2002), 2nd ed.

[18] K. F. Czaplewski and R. Q. Snurr, AIChE J. 45, 2223 (1999).

[19] J. P. Ryckaert and A. Bellemans, Faraday Discuss. Chem. Soc. 66, 95 (1978).

[20] A. G. Bezus, A.V. Kiselev, A. A. Lopatkin, and P. Q. J. Du, J. Chem. Soc., Faraday Trans. 2 74, 367 (1978).

[21] D. Dubbeldam, S. Calero, T. J. H. Vlugt, R. Krishna, T. L. M. Maesen, E. Beerdsen, and B. Smit, Phys. Rev. Lett. 93, 088302 (2004).

[22] D. Dubbeldam, S. Calero, T. J. H. Vlugt, R. Krishna, T. L. M. Maesen, and B. Smit, J. Phys. Chem. B 108, 12301 (2004).

[23] E. Beerdsen, B. Smit, and S. Calero, J. Phys. Chem. B 106, 10659 (2002). 